\title{
Return to sport and work after medial open wedge high tibial osteotomy : a case series
}

\author{
Francis De Neve, Brecht Braems, Milan Holvoet, Marie-Angélique De Scheerder, Nele Arnout, Jan Victor
}

From the Department of orthopaedic surgery and traumatology, Ghent University Hospital, Ghent, Belgium

Data on return to work and sport following open wedge high tibial osteotomy (HTO) have been underreported. Furthermore, there is no clear consensus in literature about the postoperative alignment goals following HTO. A retrospective case series was performed to evaluate return to sport and work following open wedge HTO.

The University of California, Los Angeles scale, the German classification system according to the Reichsausschuß für Arbeitszeitermittlung, the Tegner score and the Knee injury and Osteoarthritis Outcome Score were used to asses the employment status, sport status and clinical outcome at the time of surgery and at final follow-up, minimum 2 years after surgery. The pre- and postoperative hip knee ankle angle (HKA) were documented. The desired postoperative alignment target was $0^{\circ}-2^{\circ}$ valgus mechanical axis.

30 open wedge HTOs were performed of which 27 patients were retrospectively included in the study. 25 out of 26 patients returned to work and 15 out of 17 patients returned to sport following surgery. Outcome scores were significantly higher after surgery. The mean postoperative HKA was $0,9^{\circ}$ of valgus mechanical axis.

This study shows excellent outcome in sport and work activity and clinical outcome after open wedge HTO. We furthermore suggest that these outcomes can be obtained with a postoperative alignment of $0^{\circ}-2^{\circ}$ of valgus mechanical axis.

Keywords : knee surgery ; high tibial osteotomy ; return to sport ; return to work; osteoarthritis.

No benefits or funds were received in support of this study. None of the authors have a conflict of interest.

\section{INTRODUCTION}

High tibial osteotomy (HTO) is recommended for young active patients, who suffer primary degenerative arthritis of the knee joint involving a single compartment in a malaligned limb (1).

Advantages of HTO, in contrast to arthroplasty, are the preservation of the native joint and the absence of activity restriction (2).

2 recent systematic reviews $(2,3)$ report excellent outcomes after HTO. Hoorntje et al. reported that 8 out of 10 patients return to work and sport. Although they return to the same or higher workload, they report a return to lower-impact sports (3). Ekhtiari et al reported that the majority of patients return to an equal or greater level of sport and work (2).

\footnotetext{
Francis De Neve ${ }^{1,2}$,

Brecht Braems ${ }^{1}$,

Milan Holvoet ${ }^{1}$,

Marie-Angélique De Scheerder ${ }^{3}$,

Nele Arnout ${ }^{1}$,

- Jan Victor ${ }^{1}$.

${ }^{\prime}$ Department of orthopaedic surgery and traumatology, Ghent University Hospital, Ghent, Belgium.

${ }^{2}$ Department of orthopaedic surgery and traumatology, SintJozefskliniek, Izegem.

${ }^{3}$ Department of general internal medicine, Ghent University Hospital, Ghent, Belgium.

Correspondence : Francis Deneve, Ghent University Hospital, Ghent, Belgium.

Email : francis.deneve@icloud.com

- 2021, Acta Orthopædica Belgica.
} 
However the current literature presents several limitations as the different techniques in use are not distinguished (closing wedge osteotomy, opening wedge osteotomy or dome osteotomy), indications are poorly reported, surgeries are often combined, and the outcomes measurement scales are not standardized. We report a case series of patients undergoing the same surgical procedure for the same clinical indication, namely varus malalignment and medial osteoarthritis. Additionally, we retrospectively assessed if a postoperative valgus mechanical axis of 0-2 degrees is advisable. Most previous publications report a postoperative alignment of $2^{\circ}$ to $8^{\circ}$ of valgus mechanical axis following HTO (4-8). To our knowledge only more recently, some authors set the goal for postoperative alignment partially or complete below 2 degrees of valgus mechanical axis (9-11). One of these authors, La Prade et al. set the goal for postoperative alignment to a weight bearing line which crosses at a point of $56 \%$ of the width of the tibia plateau, with $0 \%$ the medial border and $100 \%$ the lateral border, which is in our experience in the range of $0-2$ degrees of valgus (11).

\section{MATERIALS AND METHODS}

This retrospective observational case series was approved by the local ethical committee (B670201630636). 30 open wedge high tibial osteotomies were performed in 30 consecutive patients between January 2011 and September 2015 at the department of Orthopedic Surgery and Traumatology of the University Hospital Ghent. All patients were operated by two senior surgeons. Inclusion criteria for the study were age (between 18-60y), symptomatic medial osteoarthritis (Kellgren-Lawrence grade 1 and 2) and varus malalignment of more than 1,5 degrees (12). Exclusion criteria for the study were active knee flexion below $120^{\circ}$, extension deficit exceeding $10^{\circ}$, instability at the time of surgery, concomitant meniscal repair/meniscal transplantation, concomitant meniscectomy, concomitant ligament repair, concomitant cartilage therapy, inflammatory arthropathy, osteochondritis dissecans and previous tibial plateau fracture.
The primary endpoint of the study was to assess the employment status and participation in sport activities at time of surgery and at final follow-up.

To evaluate the level of sport activity, workload and clinical outcome, different questionnaires were obtained preoperatively and postoperatively at final follow-up. The University of California, Los Angeles scale (UCLA) (13) was used to assess the level of physical activity. On a scale of $0-10$, the current level of activity is graded, from completely inactive to active at a regularly basis in impact sports. The German classification system according to the Reichsausschuß für Arbeitszeitermittlung (REFA) was used to classify the workload preoperatively and at final follow-up (14). On a scale of 0 to 4, the work intensity is graded, 0 being work without special physical strain and 4 being work with most heavily physical strain. The Tegner score (15) interrogates on the following subjects : recreation, competitive sports and work. It is an activity grading scale where work and sport activities are graded numerically from 0 to 10 .

The Knee injury and Osteoarthritis Outcome Score (KOOS) (16) was used for the clinical assessment preoperatively and at the time of follow-up. The questionnaire has five separately scored items : pain, function in daily living (ADL), function in sport and recreation, other symptoms, and knee-related quality of life (QOL). It consists of 42 questions and each question is assigned a score (0-4). For each subscale a normalized score is calculated (100 indicates no symptoms and 0 indicates extreme symptoms). Subanalysis was done on participants that were involved in work/sports before surgery (REFA and Tegner Adjusted).

Range of motion and tender pain were recorded preoperatively (based on the patients chart) and at the time of follow-up. Stability was assessed postoperatively. Assessing range of motion was performed using a universal goniometer (17). Knee stability was assessed by ligament examination, according the International Knee Document Committee Knee Form. Grades A, B, C or D were applied for the different tests (18).

Pre-and postoperative radiographs included full-weight-bearing long-standing anteroposterior radiographs of the whole lower extremity. The 


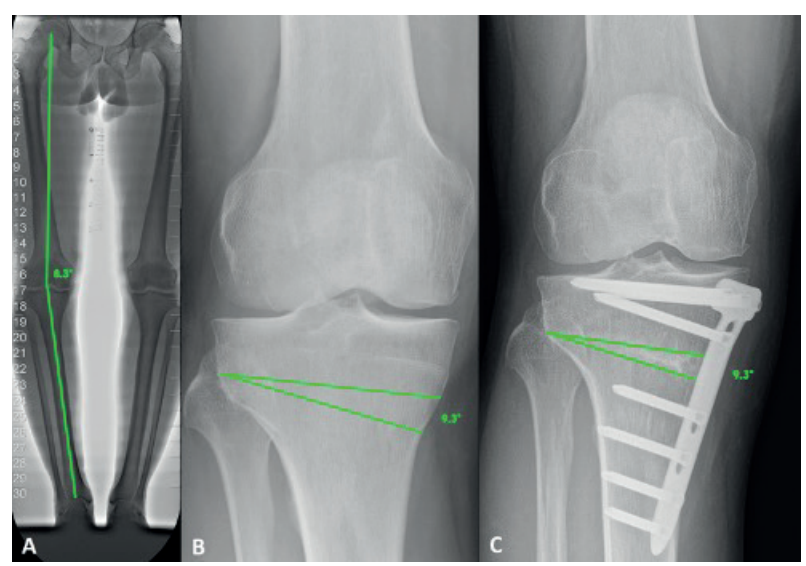

Figure 1. - Data from one of the study participants. 1A: Measurement of the HKA-angle on a full-weight-bearing longstanding AP radiograph of the entire lower extremities. 1B : The correction wedge is drawn on an AP view of the knee with appropriate correct.

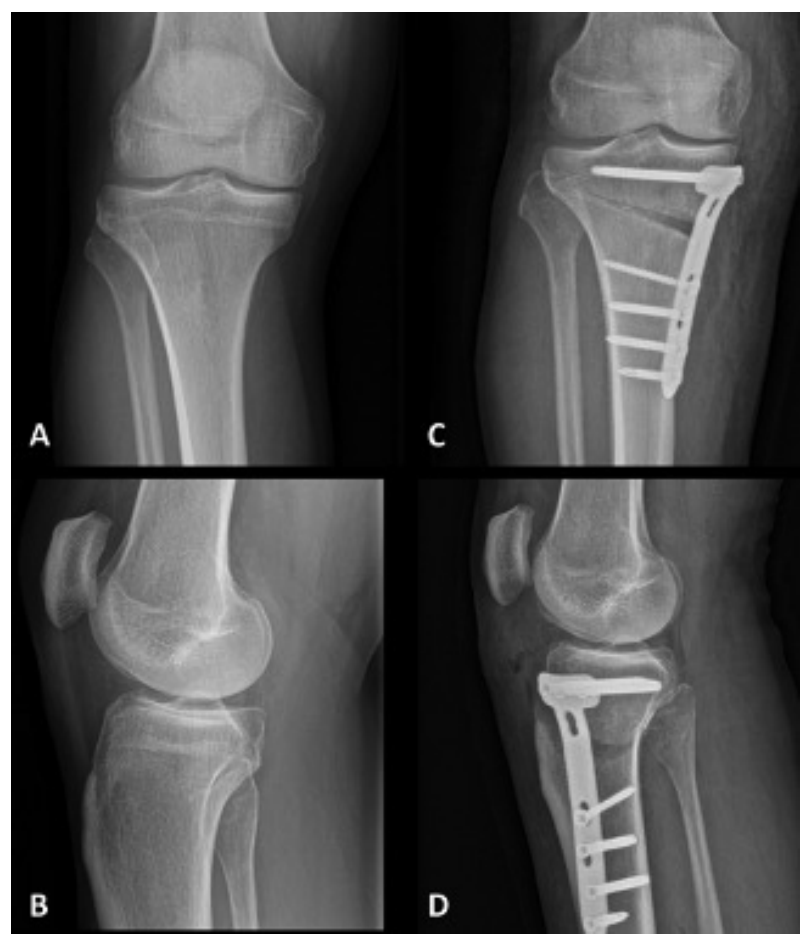

Figure 2. - Data from one of the study participants. 2A : Preoperative AP radiograph. 2B : Preoperative lateral radiograph. 2C : Postoperative AP radiograph. 2D : Postoperative lateral radiograph.

participants were positioned with the tibial tuberosity facing the x-ray beam and with the knee in $0^{\circ}$ of flexion.
The pre-and postoperative hip knee ankle angle (HKA) were documented by one experienced senior surgeon. The point on the tibia plateau where the weight bearing line (WBL) crosses the tibia plateau was documented. The point on the tibia plateau where the WBL crosses the tibia plateau was expressed as percentage of the width of the tibia plateau (\%WBL), with $0 \%$ the medial border and $100 \%$ the lateral border. The HKA and the point on the tibia plateau where the WBL crosses the tibia plateau was used to assess the alignment pre-and postoperatively.

The goal of postoperative alignment was to achieve a postoperative HKA angle of $0^{\circ}-2^{\circ}$ of valgus. To achieve this goal we aimed for a postoperative alignment of $1^{\circ}$ of valgus mechanical axis. First, the HKA is measured on a full leg weight-bearing radiograph. This angle plus $1^{\circ}$ of overcorrection represents the correction angle of the wedge. Secondly, the correction wedge, with appropriate correction angle, is drawn on a standard AP view of the knee at the desired location (fig. 1).

Patients were treated with open wedge osteotomy mainly according to the surgical technique by Lobenhoffer (19), in which the Tomofix Medial High Tibial Plate system (Tomofix MHT, De Puy Synthes) is applied to stabilize the osteotomy without the use of bone grafts to fill the osteotomy gap (19). Figure 2 shows representative preoperative and postoperative radiographs.

\section{Statistical analysis}

IBM SPSS statistics software version 23 (SPSS Inc, Chicago, IL) for windows was used for statistical analysis. The results of the KOOS, Tegner and UCLA scores were compared (preoperatively and postoperatively) with the paired Student's t test, normal distribution and equality of variances was present. Normality of distribution was estimated by Shapiro-Wilk test. Equality of variances was tested by Levene's test. The results of the REFA score, the radiographic findings and the ROM were compared (preoperatively and postoperatively) with the nonparametric Wilcoxon rank test, because of a non-normal distribution. The level of significance was set to $\mathrm{p}<0.05$. 


\section{RESULTS}

30 open wedge HTOs were performed in 30 consecutive patients. 27 patients were included (fig. 3 ). One patient was excluded because of instability due to rupture of the ACL and another because of severe tibial plateau fracture in the past history. One patient was lost to follow-up. No patients were converted to a total knee arthroplasty and no patients had revision surgery.

The patient's characteristics are presented in table I. Patients were invited for one follow-up visit with a minimum interval of 2 years after the surgery. Mean follow-up was 32.2 [24.7;39.7] months. Only 1 patient showed obesity. All patients suffered from medial osteoarthrosis with mean malalignment of $6.1^{\circ}[3.6 ; 8.6]$ of varus mechanical axis.

\section{Sport and activity scores}

Before surgery $26(96.3 \%)$ patients were employed, compared to $25(92.6 \%)$ at final followup. One patient did not return to work, due to knee impairment. Before surgery $17(63.0 \%)$ patients participated in sport activities on a regular basis, compared to $20(74.1 \%)$ patients at final follow-up. Of all patients who participated in sport activities before surgery, only two patients did not return to sport. 10 patients did not participate in sport activities before surgery, 5 of them reinitiated sport activities after surgery. After surgery the

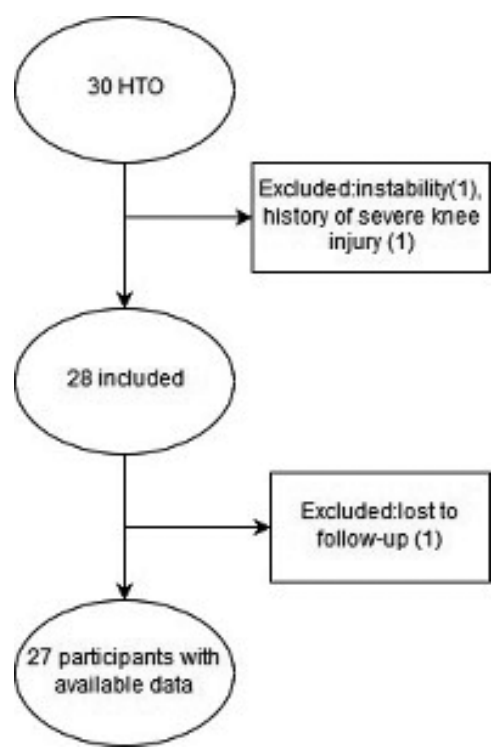

Figure 3. - Flowchart of the study series.

Table I. - Demographic Data of the series

\begin{tabular}{|l|c|}
\hline \multicolumn{2}{|c|}{ Data } \\
\hline Number of participants (N) & 27 \\
\hline Gender (Male/Female) & M:23; F:4 \\
\hline Side (Left/Right) & L:17; R:10 \\
\hline $\begin{array}{l}\text { Age at evaluation in years, } \\
\text { mean[95\%CI] }\end{array}$ & $41.2[31.6 ; 50.8]$ \\
\hline $\begin{array}{l}\text { Follow-up period in months, } \\
\text { mean[95\%CI] }\end{array}$ & $32.2[24.7 ; 39.7]$ \\
\hline BMI in kg/m², mean[95\%CI] & $25.4[22.6 ; 28.2]$ \\
\hline
\end{tabular}

Table II. - Overview of the reported outcomes

\begin{tabular}{|l|c|c|c|}
\hline & pre-operative $[95 \% \mathrm{CI}]$ & post-operative[95\%CI] & -value \\
\hline UCLA & $4.7[2.7 ; 6.7]$ & $7.2[5.3 ; 9.1]$ & $<0.001$ \\
\hline REFA & $1.8[0.9 ; 2.7]$ & $1.8[0.9 ; 2.7]$ & 0.317 \\
\hline REFA adjusted & $2.2[1.4 ; 3.0]$ & $2.2[1.4 ; 3.0]$ & 0.317 \\
\hline Tegner & $3.0[1.5 ; 4.5]$ & $3.9[1.9 ; 5.9]$ & $<0.001$ \\
\hline Tegner adjusted & $4.2[3.2 ; 5.2]$ & $5.3[3.6 ; 7.0]$ & 0.014 \\
\hline ROM & $0.5^{\circ}[-0.8 ; 1.8]-126.8^{\circ}[118.3 ; 135.3]$ & $0.40^{\circ}[-1.0 ; 1.8]-134.8^{\circ}[131.4 ; 138.2]$ & 0.010 \\
\hline Koos Sport and Rec & $39.7[26.5 ; 52.9]$ & $66.0[38.2 ; 93.8]$ & $<0.001$ \\
\hline Koos QoL & $40.1[22.7 ; 57.5]$ & $66.1[45.6 ; 86.6]$ & $<0.001$ \\
\hline$\%$ WBL & $22.1[13.2 ; 31.0]$ & $53.4[43.4 ; 63.4]$ & $<0.001$ \\
\hline HKA & $6.1^{\circ}[3.6 ; 8.6]$ & $-0.9^{\circ}[-3.0 ; 1.2]$ & $<0.001$ \\
\hline
\end{tabular}


UCLA and Tegner scores increased significantly compared to pre-operative measures $(\mathrm{p}<0.001)$. The postoperative REFA score did not increase significantly $(\mathrm{p}=0.317)$. Results of all outcome measures are summarized in Table II. After surgery the preoperative subscales Sport/rec and QoL scores increased significantly $(\mathrm{p} \leq 0.001)$.

\section{Clinical outcomes}

A significant change in flexion postoperatively $134,8^{\circ}[131.4 ; 138.2]$ compared to preoperatively $126,8^{\circ}[118.3 ; 135.3]$ was observed $(p=0,025)$. Preoperative joint effusion was present in $12(44.4 \%)$ patients. At final follow-up $4(15.0 \%)$ patients showed joint effusion, in all these cases the effusion was slight. $21(77.8 \%)$ patients showed tender pain preoperatively compared to only $5(18.5 \%)$ at final follow-up. All patients showed a stable knee joint at final follow-up.

\section{Radiographic outcomes}

Preoperatively the mean HKA-angle was $6.1^{\circ}$ [3.6;8.6] of varus mechanical axis, postoperatively the mean HKA-angle was $0.9^{\circ}$ [-1.2;3.0] of valgus mechanical axis. This change was significant $(p \leq 0.001)$. Preoperatively the mean WBL crossed at the tibia plateau at $22,1 \%[13.2 ; 31.0]$ of the width of the tibia plateau, at final follow-up the mean WBL crossed at the tibia plateau at $53,4 \%[43.4 ; 63.4]$ of the width of the tibia plateau. This increase was significant with $\mathrm{p} \leq 0.001$.

\section{Complications and plate removal}

4 out of $27(14.8 \%)$ participants presented a complication; there were two cases of cellulitis that were treated with oral antibiotics, one case of wound dehiscence that resolved with aseptic wound care and one case of postoperative hematoma. In 13 (48.1\%) patients hardware was removed because of hardware irritation,. 2 patients had an additional intervention : a resection of the proximal tibiofibular joint and an arthroscopic debridement of the medial compartment because of locking due to unstable osteochondral fragment.

\section{DISCUSSION}

This study shows that the majority of patients with medial osteoarthritis and varus malalignment return to work and sport activities following open wedge HTO. The mean level of sport activity was higher and the mean level of workload was equal after surgery compared to before surgery.

These results are in line with previous reports. Saragaglia et al. ${ }^{20}$ reported a return to sport of $85,5 \%$ in a consecutive series of 83 open wedge osteotomies and Salzmann et al. (21) published a similar series of 65 open wedge osteotomies and reported that at the time of survey $90.9 \%$ of the patients were active in sport, compared to $87.9 \%$ before surgery. Both studies however had less restrictive inclusion criteria and showed less uniformity in the evaluation strategies, possibly explaining the somewhat lower outcomes.

In a prospective follow-up study of 64 patients with follow-up of 24 months Saier et al. (22) reported a return to work rate of $90 \%$ following open wedge HTO without any limitations. Only the medial open wedge technique was used. This return to work rate is very comparable to the return to work rate we reported, being $96.2 \%$. In a recent single-center, retrospective study of 30 patients who underwent medial open wedge HTO for medial osteoarthritis Bastard et al. showed a return to sports rate of $100 \%$, $73,3 \%$ of the patients at the same level, $23,3 \%$ at a higher level and 3,4\% at a lower level (23). In our study $88.2 \%$ of patients who participated in sport activities before surgery continued to do so. In the study published by Bastard et al. the postoperative Tegner score did not increase compared to the preoperative values, in contrast to our study results showing a significant increase in the mean level of sport activities. Important to mention is the short follow-up period of only 1,3 years in the study of Bastard et al, the mean follow up in our study was 2,7 years with a minimum follow up period of 2 years.

The survival rate in terms of TKP or UKP was $100 \%$ with a minimum follow-up of 2 years. Further follow-up is necessary to see if these results are sustainable as other studies have shown an expected decrease in survival rate over time related 
to progressive osteoarthritis disease and aging (24). It has been shown that a higher BMI is related to a lower survival rate, one patient in our study showed obesity which might affect survival rate in the future (25).

Besides the clinical outcome, we took advantage of this study to address the disparate data related to the postoperative alignment goals. The degree of correction is variable and controversial and there is no clear consensus about the desired postoperative alignment in patients with medial osteoarthritis of the knee (Kellgren-Lawrence grade 1 and 2) and varus malalignment. Different targets have been set for postoperative alignment. Most authors seem to believe in overcorrection of $2^{\circ}$ to $8^{\circ}$ of valgus mechanical axis. Although these publications date from the eighties-nineties, they are still com-monly referred to and applied in more recent reports (4-8). Only more recently, some authors aim for a postoperative mechanical axis completely or partially below 2 degrees of valgus mechanical axis (9-11,26). Although the shift in literature suggests that there is a return from overcorrection when comparing older and more recent studies, there is little evidence supporting the clinical benefits of a slight postoperative mechanical valgus compared to more conservative correction aims. Feucht et al. (9), McNamara et al. (10) and La Prade et al. (11) all reported good clinical outcome but only La Prade et al. quantified the outcome with a modified Cincinnati Knee Scores which improved significantly from 42.9 preoperatively to 65.1 at a mean of 3.6 years of follow-up.

The target in the present study was $0^{\circ}-2^{\circ}$ of valgus. To achieve this we aimed for a postoperative HKA of $1^{\circ}$ of valgus. We aimed for a slight less postoperative valgus mechanical axis in order to mechanically realign the leg and unload the medial compartment without the risk of overloading the lateral compartment, gradual medial collateral ligament failure, progressive valgus deformity or creating a cosmetically unappealing valgus. Dugdale et al. shared some of these concerns of overcorrection ${ }^{7}$ and in a study of Hernigou et al. (5) all knees with a postoperative HKA of more than $6^{\circ}$ of valgus mechanical axis showed progressive degenerative changes in the lateral compartment. Overall, literature addressing the subject of overcorrection is sparse and outdated. Biomechanical studies support that the medial compartment is unloaded even in neutral or slight valgus mechanical alignment. Agneskircher et al. and Riegger Kruch et al. conclude in two in vitro biomechanical studies that even neutral mechanical alignment leads to decreased loading of the medial compartment and suggest that markedly overcorrection might not be necessary $(27,28)$. Mina et al. found in a laboratory study that contact pressure is approximately equally distributed between the medial and lateral compartments for alignments of $0^{\circ}-4^{\circ}$ of anatomical valgus (29). Furthermore, we believe as described by Saragaglia et al. that a varus knee or a knee with a neutral axis is the best for participating in running sports (20). When the knee is too much overcorrected, the athlete may struggle to regain their proprioception and ground support which will impair return to sport to the previous level, even though a residual varus might imply a higher risk of residual pain when participating in running sport.

In our study only patients with medial osteoarthritis (Kellgren-Lawrence grades 1 and 2) were selected, and we found excellent clinical and radiographical results with a postoperative alignment goal of $0-2^{\circ}$ of valgus mechanical axis after a minimum of 2 years follow-up. Unfortunately, we can only suggest but not correlate the excellent clinical outcomes with the correction angle, as the study design does not allow us to draw such conclusions.

As discussed above there are several limitations to our study. We present data from a small case series of 27 patients. The study is observational and retrospective which due to its design is vulnerable to selection bias. As the study lacks a comparator group it is difficult to validate this data and show a cause-effect. Furthermore, the follow-up period is too short to inform on long-term outcome.

\section{CONCLUSION}

This study confirms that the majority of patients with medial osteoarthritis of the knee (KellgrenLawrence grades 1-2) and varus malalignment return to sport and work after open wedge HTO and 
that the mean level of sport activity is higher after surgery than before. Furthermore, we observed these excellent clinical results with a mean postoperative valgus mechanical axis of $0,9^{\circ}$. We acknowledge that longer follow-up is needed to make any longterm conclusion concerning outcome and that our study design does not allow conclusions on posoperative alignment. Larger cohorts with predefined alignment goals and blinded controls are needed to further enlight on these findings.

\section{REFERENCES}

1. DeMeo PJ, Johnson EM, Chiang PP, Flamm AM, Miller MC. Midterm follow-up of opening-wedge high tibial osteotomy. The American journal of sports medicine 2010 ; 38(10) : 2077-84.

2. Ekhtiari S, Haldane CE, de Sa D, Simunovic N, Musahl V, Ayeni OR. Return to Work and Sport Following High Tibial Osteotomy: A Systematic Review. J. Bone Joint Surg. Am. 2016 ; 98(18) : 1568-77.

3. Hoorntje A, Witjes S, Kuijer P, et al. High Rates of Return to Sports Activities and Work After Osteotomies Around the Knee : A Systematic Review and Meta-Analysis. Sports Med. 2017 ; 47(11) : 2219-44.

4. Coventry MB, Ilstrup DM, Wallrichs SL. Proximal tibial osteotomy. A critical long-term study of eighty-seven cases. JBJS 1993 ; 75(2) : 196-201.

5. Hernigou P, Medevielle D, Debeyre J, Goutallier D. Proximal tibial osteotomy for osteoarthritis with varus deformity. A ten to thirteen-year follow-up study. J. Bone Joint Surg. Am. 1987 ; 69(3) : 332-54.

6. Insall JN, Joseph DM, Msika C. High tibial osteotomy for varus gonarthrosis. A long-term follow-up study. JBJS $1984 ; 66(7): 1040-8$.

7. Dugdale TW, Noyes FR, Styer D. Preoperative planning for high tibial osteotomy. The effect of lateral tibiofemoral separation and tibiofemoral length. Clin. Orthop. Relat. Res. 1992 ; (274) : 248-64.

8. Miniaci A, Ballmer FT, Ballmer PM, Jakob RP. Proximal tibial osteotomy. A new fixation device. Clin. Orthop. Relat. Res. 1989 ; (246) : 250-9.

9. Feucht MJ, Minzlaff P, Saier T, et al. Degree of axis correction in valgus high tibial osteotomy : proposal of an individualised approach. Int. Orthop. $2014 ; 38(11)$ : 227380.

10. McNamara I, Birmingham TB, Fowler PJ, Giffin JR. High tibial osteotomy : evolution of research and clinical applications--a Canadian experience. Knee Surg. Sports Traumatol. Arthrosc. 2013 ; 21(1) : 23-31.

11. LaPrade RF, Spiridonov SI, Nystrom LM, Jansson KS. Prospective outcomes of young and middle-aged adults with medial compartment osteoarthritis treated with a proximal tibial opening wedge osteotomy. Arthroscopy : The Journal of Arthroscopic \& Related Surgery 2012 ; 28(3) : 354-64.

12. Kellgren JH, Lawrence JS. Radiological assessment of osteo-arthrosis. Annals of the rheumatic diseases 1957 ; 16(4) : 494-502.

13. Zahiri CA, Schmalzried TP, Szuszczewicz ES, Amstutz HC. Assessing activity in joint replacement patients. The Journal of arthroplasty 1998 ; 13(8) : 890-5.

14. REFA. http :/www.refa.de/service/wir/refabundesverband.

15. Tegner Y, Lysholm J. Rating systems in the evaluation of knee ligament injuries. Clinical orthopaedics and related research 1985 ; (198) : 43-9.

16. Roos EM, Roos HP, Lohmander LS, Ekdahl C, Beynnon BD. Knee Injury and Osteoarthritis Outcome Score (KOOS)-development of a self-administered outcome measure. J. Orthop. Sports Phys. Ther. 1998 ; 28(2) : 88-96.

17. CC Norkin DW. Techniques and procedures in Measurement of joint motion : A guide to goniometry : FA Davis Company, Philadelpha ; 2009.

18. Hefti F, Müller W, Jakob RP, Stäubli HU. Evaluation of knee ligament injuries with the IKDC form. Knee Surg. Sports Traumatol. Arthrosc. 1993 ; 1(3-4) : 226-34.

19. Staubli AE, De Simoni C, Babst R, Lobenhoffer P. TomoFix : a new LCP-concept for open wedge osteotomy of the medial proximal tibia - early results in 92 cases. Injury 2003 ; 34 Suppl 2 : B55-62.

20. Saragaglia D, Rouchy RC, Krayan A, Refaie R. Return to sports after valgus osteotomy of the knee joint in patients with medial unicompartmental osteoarthritis. International Orthopaedics 2014.

21. Salzmann GM, Ahrens P, Naal FD, et al. Sporting activity after high tibial osteotomy for the treatment of medial compartment knee osteoarthritis. American Journal of Sports Medicine 2009 ; 37(2) : 312-8.

22. Saier T, Minzlaff P, Feucht MJ, et al. Health-related quality of life after open-wedge high tibial osteotomy. Knee Surg. Sports Traumatol. Arthrosc. 2015.

23. Bastard C, Mirouse G, Potage D, et al. Return to sports and quality of life after high tibial osteotomy in patients under 60 years of age. Orthop. Traumatol. Surg. Res. 2017 ; 103(8) : 1189-91.

24. Annette W, Toksvig-Larsen S, Lindstrand A. Ten-year results of physical activity after high tibial osteotomy in patients with knee osteoarthritis. Knee Surgery, Sports Traumatology, Arthroscopy 2015 : 1-8.

25. Akizuki S, Shibakawa A, Takizawa T, Yamazaki I, Horiuchi H. The long-term outcome of high tibial osteotomy : a ten- to 20-year follow-up. J. Bone Joint Surg. Br. 2008 ; 90(5) : 592-6.

26. Muller M, Strecker W. Arthroscopy prior to osteotomy around the knee? Arch. Orthop. Trauma Surg. 2008; 128(11) : 1217-21.

27. Agneskirchner JD, Hurschler C, Wrann CD, Lobenhoffer P. The effects of valgus medial opening wedge high tibial osteotomy on articular cartilage pressure 
of the knee : a biomechanical study. Arthroscopy 2007 ; $23(8): 852-61$.

28. Riegger-Krugh C, Gerhart TN, Powers WR, Hayes

WC. Tibiofemoral contact pressures in degenerative joint disease. Clin. Orthop. Relat. Res. 1998 ; (348) : 233-45.
29. Mina C, Garrett WE, Jr., Pietrobon R, Glisson R, Higgins L. High tibial osteotomy for unloading osteochondral defects in the medial compartment of the knee. Am. J. Sports Med. 2008 ; 36(5) : 949-55. 